\title{
Computed tomography as a predictor of the extent of the disease and surgical outcomes in ovarian cancer
}

\author{
Zeynep Ceren Cerci ${ }^{1}$, Derya Kilic Sakarya ${ }^{2}$, Mehmet Hakan Yetimalar ${ }^{3}$, Incim Bezircioglu ${ }^{3}$, \\ Burcu Kasap ${ }^{4}$, Emre Baser ${ }^{5}$, Kamil Yucel ${ }^{6}$ \\ 'Department of Obstetrics and Gynecology, Sandıklı State Hospital, Afyon, Turkey \\ ${ }^{2}$ Department of Obstetrics and Gynecology, Konya Training and Research Hospital, Turkey \\ ${ }^{3}$ Department of Obstetrics and Gynecology, Izmir Katip Celebi University Ataturk Training and Research Hospital, Izmir, Turkey \\ ${ }^{4}$ Department of Obstetrics and Gynecology, Mugla Sitki Kocman University School of Medicine, Mugla, Turkey \\ ${ }^{5}$ Department of Obstetrics and Gynecology, Etlik Zubeyde Hanim Women's Health Teaching and Research Hospital, Ankara, Turkey \\ ${ }^{6}$ Department of Radiology, Izmir Katip Celebi University Ataturk Training and Research Hospital, Izmir, Turkey
}

\begin{abstract}
Objectives: The aim of the present study is to determine the predictive value of Computed Tomography (CT), alone or in combination with serum CA-125 levels, for preoperative staging, detection of the extent of the disease, and surgical complications in patients with ovarian carcinoma.
\end{abstract}

Material and methods: One hundred and fourteen patients diagnosed with ovarian carcinoma following an exploratory laparotomy with a preoperative CT scan, performed between January 2007 and June 2013, were enrolled in the study. Preoperative CT and intraoperative surgical findings were compared using 14 parameters and predictions of CT for gastrointestinal, genitourinary, and cardiovascular complications. All radiological features and clinical characteristics were analyzed statistically.

Results: CT and surgical findings correlated (sensitivity/ specificity) as follows: uterine and tubal spread (66\%/89\%), cervical involvement (100\%/80\%), peritoneal nodulesincreased density-carcinomatosis (57\%/93\%), omental involvement (68\%/95\%), retroperitoneal involvement (25\%/84\%), ascites (85\%/87\%), perirectal and perivesical fat plan obliteration (43\%/94\%), liver metastasis (50\%/91\%), small and large bowel involvement (47\%/95\%), adnexal mass (94\%/70\%), and other metastases (47\%/86\%). Also, CT findings were found to be statistically insignificant for prediction of mesenteric involvement, bladder metastasis, and diaphragmatic involvement. The overall CT sensitivity and specificity at detecting intraoperative findings was $91 \%$ and $71 \%$, respectively. We found a statistically significant correlation between intestinal involvement on CT and the necessity of additional surgical procedures.

Conclusions: CT is a widely used imaging method in the preoperative evaluation of ovarian cancer. However, its predictive value, sensitivity and specificity differ, depending on the anatomical region.

Key words: computed tomography, complication, prediction, ovarian carcinoma, cytoreductive surgery, radiologic imaging

Ginekologia Polska 2016; 87, 5: 326-332

\section{INTRODUCTION}

Ovarian cancer (OC) is the most lethal among all gynecological malignancies, with the overall lifetime risk of developing cancer estimated at 1.8\% [1]. Despite years of $\mathrm{OC}$ research, the mortality rates could not be reduced. Unfortunately, over $70 \%$ of the affected patients are diagnosed at advanced stages of the disease and long-term survival rates are quite low (10-30\%) [2]. Accurate determination of the disease spread during the preoperative period will result in maximum benefit from the treatment by providing the necessary conditions for optimal debulking. In addition, it is a well-known fact that surgical debulking is the cornerstone of therapy and that minimal residual disease after initial surgery is the most important factor in increasing chemo- 
therapy response, and consequently the survival rates, for women with OC $[3,4]$.

Various radiological and serological diagnostic methods are used to identify high-risk patients, to term peri-operative complications, to predict optimal cytoreduction, and to identify patients who may benefit from the neo-adjuvant therapy. However, none of the methods proved to have sufficient predictive value [5-7]. Due to reasonable costs and wide availability, Computed Tomography (CT) is one of the most commonly used imaging methods and CA-125 is the most studied serological marker in $\mathrm{OC}$ for this purpose [8-10]. Preoperative CT scans can identify the presence of the disease in anatomic regions which are difficult to resect, i.e. perirectal-perivesical fat plan, bladder, retroperitoneal lymph node, small and large intestines, diaphragm, lesser sac, liver, mesentery, adenopathy above the renal vessels, etc.). Nevertheless, as might be expected, it does not always yield the same quality $[5,9,11]$.

In our study, we aimed to determine the sensitivity and specificity of CT, alone or in combination with serum CA-125 levels, to detect the spread of the disease based on the organs.

\section{MATERIAL AND METHODS}

The study was performed retrospectively in the cooperation of the Gynecology-Oncology and Radiology Departments at Katip Celebi University and Izmir Ataturk Teaching and Research Hospital. A total of 114 patients (aged 28-91) diagnosed with ovarian carcinoma following an exploratory laparotomy with a preoperative CT scan between January 2007 and June 2013, were enrolled. Laparotomy was performed in all patients by at least two experts in the field of oncological gynecology. The surgical procedures included: cytological sampling, if there is a uterus; total abdominal hysterectomy, bilateral/unilateral salpingo-oophorectomy, partial/total omentectomy, pelvic and para-aortic lymph node dissection and, where necessary, maximum tumor resection in order to do primary cytoreductive surgery. Only the patients with the preoperative CT scans of the pelvis and abdomen with oral and iv contrasts performed 4 weeks prior to primary surgery at the latest were included in the study. Surgical stages of all the patients according to FIGO (International Federation of Obstetrics and Gynecology) based on intraoperative findings and histopathological data were recorded. Preoperative staging was done by evaluation of CT examinations based on FIGO staging system. Preoperative CT scans were re-reviewed by one radiologist, who was blinded to the previous surgical outcomes. Patient serum CA-125 levels were analyzed by the Department of Biochemistry using the chemiluminescence method, with normal values between 0-35 IU/mL. Testing for CA-125 levels was performed within 4 weeks prior to surgery at the latest.
Preoperative $\mathrm{CT}$ findings and intraoperative surgical findings were compared for 14 parameters. These parameters were: adnexal mass, depiction, presence of ascites, uterine and tubal invasion, cervical invasion, perirectal-perivesical fat plan obliteration, bladder metastasis, peritoneal nodules-diffuse peritoneal thickening, omental involvement, retroperitoneal lymph node involvement, the involvement of small and large intestine, mesenteric involvement, diaphragmatic involvement, liver involvement, and other respectively.

According to the serum CA-125 levels, compliance of the CT findings with intraoperative findings was investigated and preoperative CT findings were compared with gastrointestinal system (GIS), genitourinary system (GUS) and cardiovascular system (CVS) complications and additional surgeries respectively.

Statistical analysis was performed using SPSS 15.0 for Windows (SPSS Inc, Chicago, III, USA) statistical software. Categorical variables were described using frequency distribution. Receiver Operating Characteristic plots (ROC) were graphed and the Area Under the Curve (AUC) was calculated for each categorical variable. Sensitivity and specificity were calculated based on the ROC analysis. Wilcoxon signed rank analyses was used to compare preoperative and post-operative stages. The $p$-value of $<0.05$ was considered as statistically significant.

\section{RESULTS}

Mean patient age was 59 years (range 28-91). Histopathological characteristics of the patients were summarized in Table 1. Preoperative serum CA-125 levels ranged from $5 \mathrm{U} / \mathrm{mL}$ to $6716 \mathrm{U} / \mathrm{mL}$. Twenty-one (21\%) patients were FIGO stage 1, 4 (4\%) were stage 2, 47 (41\%) were stage 3, and 39 (34\%) were stage 4 surgically.

We compared 14 parameters depicting the extent of the disease across preoperative $\mathrm{CT}$ findings and surgical findings. CT sensitivity and specificity for each parameter were calculated and are shown in Table 2.

According to the ROC analysis, uterine and tubal spread was found in 55 patients intraoperatively. In 36 of them these conditions could be detected correctly by preoperative CT. Forty-one patients did not have uterine and tubal

\begin{tabular}{|l|c|c|}
\hline Table 1. Histopathological range & \multicolumn{2}{l|}{} \\
\hline Histopathological type & $\mathbf{n}$ & $\%$ \\
\hline Epithelial & 98 & 86.0 \\
\hline Germ cell & 3 & 2.6 \\
\hline Metastatic & 10 & 8.8 \\
\hline Sex-cord stromal & 3 & 2.6 \\
\hline & 114 & 100.0 \\
\hline
\end{tabular}


Table 2. The compliance of intraoperative findings and CT findings according to ROC analysis

\begin{tabular}{|c|c|c|c|c|c|c|c|c|c|c|c|}
\hline & \multirow{3}{*}{ Ст } & \multicolumn{4}{|c|}{ Intraoperative } & \multirow{2}{*}{\multicolumn{2}{|c|}{ Total }} & \multirow{3}{*}{ Sensitivity } & \multirow{3}{*}{ Specificity } & \multirow{3}{*}{ AUC } & \multirow{3}{*}{$\begin{array}{c}\text { AUC } \\
\mathbf{p}\end{array}$} \\
\hline & & \multicolumn{2}{|c|}{+} & \multicolumn{2}{|c|}{-} & & & & & & \\
\hline & & $\mathbf{n}$ & $\%$ & $\mathbf{n}$ & $\%$ & n & $\%$ & & & & \\
\hline \multirow{3}{*}{ Uterine-fallopian involvement } & + & 36 & 65.5 & 7 & 17.1 & 43 & 44.8 & \multirow{3}{*}{65.5} & \multirow{3}{*}{82.9} & \multirow{3}{*}{0.742} & \multirow{3}{*}{0.001} \\
\hline & - & 19 & 34.5 & 34 & 82.9 & 53 & 55.2 & & & & \\
\hline & Total & 55 & 57.3 & 41 & 42.7 & 96 & 100.0 & & & & \\
\hline \multirow{3}{*}{ Cervical involvement } & + & 5 & 100.0 & 5 & 20.0 & 10 & 33.3 & \multirow{3}{*}{100.0} & \multirow{3}{*}{80.0} & \multirow{3}{*}{0.900} & \multirow{3}{*}{0.005} \\
\hline & - & - & - & 20 & 80.0 & 20 & 66.7 & & & & \\
\hline & Total & 5 & 16.7 & 25 & 83.3 & 30 & 100.0 & & & & \\
\hline \multirow{3}{*}{ Peritoneal carcinomatosis } & + & 35 & 57.4 & 4 & 7.8 & 39 & 34.8 & \multirow{3}{*}{57.4} & & & \\
\hline & - & 26 & 42.6 & 47 & 92.2 & 73 & 65.2 & & 92.2 & 0.748 & 0.001 \\
\hline & Total & 61 & 54.5 & 51 & 45.5 & 112 & 100.0 & & & & \\
\hline & + & 36 & 67.9 & 3 & 5.2 & 39 & 35.1 & & & & \\
\hline Omental involvement & - & 17 & 32.1 & 55 & 94.8 & 72 & 64.9 & 67.9 & 94.8 & 0.814 & 0.001 \\
\hline & Total & 53 & 47.7 & 58 & 52.3 & 111 & 100.0 & & & & \\
\hline & + & 11 & 25.0 & 7 & 15.2 & 18 & 20.0 & & & & \\
\hline Retroperitoneal involvement & - & 33 & 75.0 & 39 & 84.8 & 72 & 80.0 & 25.0 & 84.8 & 0.549 & 0.424 \\
\hline & Total & 44 & 48.9 & 46 & 51.1 & 90 & 100.0 & & & & \\
\hline & + & 52 & 85.2 & 7 & 13.5 & 59 & 52.2 & & & & \\
\hline Ascites & - & 9 & 14.8 & 45 & 86.5 & 54 & 47.8 & 85.2 & 86.5 & 0.859 & 0.001 \\
\hline & Total & 61 & 54.0 & 52 & 46.0 & 113 & 100.0 & & & & \\
\hline & + & 23 & 42.6 & 2 & 3.6 & 25 & 22.9 & & & & \\
\hline Perivesical-perirectal fat & - & 31 & 57.4 & 53 & 96.4 & 84 & 77.1 & 42.6 & 96.4 & 0.695 & 0.001 \\
\hline & Total & 54 & 49.5 & 55 & 50.5 & 109 & 100.0 & & & & \\
\hline & + & - & - & 1 & 1.2 & 1 & 1.0 & & & & \\
\hline Diaphragmatic involvement & - & 20 & 100.0 & 80 & 98.8 & 100 & 99.0 & 0.0 & 98.8 & 0.494 & 0.832 \\
\hline & Total & 20 & 19.8 & 81 & 80.2 & 101 & 100.0 & & & & \\
\hline & + & 15 & 50.0 & 7 & 9.1 & 22 & 20.6 & & & & \\
\hline Liver involvement & - & 15 & 50.0 & 70 & 90.9 & 85 & 79.4 & 50.0 & 90.9 & 0.705 & 0.001 \\
\hline & Total & 30 & 28.0 & 77 & 72.0 & 107 & 100.0 & & & & \\
\hline & + & 7 & 31.8 & 9 & 11.3 & 16 & 15.7 & & & & \\
\hline Bladder involvement & - & 15 & 68.2 & 71 & 88.8 & 86 & 84.3 & 31.8 & 88.8 & 0.603 & 0.141 \\
\hline & Total & 22 & 21.6 & 80 & 78.4 & 102 & 100.0 & & & & \\
\hline & + & 22 & 46.8 & 3 & 4.9 & 25 & 23.1 & & & & \\
\hline Small and large bowel & - & 25 & 53.2 & 58 & 95.1 & 83 & 76.9 & 46.8 & 95.1 & 0.709 & 0.001 \\
\hline & Total & 47 & 43.5 & 61 & 56.5 & 108 & 100.0 & & & & \\
\hline & + & 12 & 24.5 & 4 & 7.7 & 16 & 15.8 & & & & \\
\hline Mesenteric involvement & - & 37 & 75.5 & 48 & 92.3 & 85 & 84.2 & 24.5 & 92.3 & 0.584 & 0.146 \\
\hline & Total & 49 & 48.5 & 52 & 51.5 & 101 & 100.0 & & & & \\
\hline & + & 9 & 47.4 & 13 & 13.7 & 22 & 19.3 & & & & \\
\hline Other distant metastasis & - & 10 & 52.6 & 82 & 86.3 & 92 & 80.7 & 47.4 & 86.3 & 0.668 & 0.021 \\
\hline & Total & 19 & 16.7 & 95 & 83.3 & 114 & 100.0 & & & & \\
\hline & + & 88 & 93.6 & 3 & 30.0 & 91 & 87.5 & & & & \\
\hline Adnexal mass & - & 6 & 6.4 & 7 & 70.0 & 13 & 12.5 & 93.6 & 70.0 & 0.818 & 0.001 \\
\hline & Total & 94 & 90.4 & 10 & 9.6 & 104 & 100.0 & & & & \\
\hline
\end{tabular}

CT — computed tomography, AUC — area under curve 
spread, and in 7 of them CT yielded false-positive results (66\% sensitivity, $89 \%$ specificity). Cervical involvement could be detected intraoperatively with $\mathrm{CT}$ in all of the 5 affected patients. However, 5 of the 25 patients without cervical involvement had false-positive results on CT (100\% sensitivity, $80 \%$ specificity). Peritoneal nodules-diffuse peritoneal thickening was found in 61 patients intraoperatively and CT could detect 35 of these patients correctly. Four patients without peritoneal nodules had false-positive results on preoperative CT ( $57 \%$ sensitivity, 93\% specificity). Thirty-six of the 53 patients with omental involvement could be identified correctly with $\mathrm{CT}$, but 3 of the 51 patients without omental involvement had false-positive results on CT (68\% sensitivity, $95 \%$ specificity). CT detected just 11 of the 44 patients with intraoperatively and histopathologically proven retroperitoneal involvement (iliac, obturator, para-aortic involvement), and yielded false-positive results in 7 of the 46 patients without retroperitoneal involvement ( $25 \%$ sensitivity). Ascites was determined correctly by CT in 52 of the 61 patients. However, CT also yielded false-positive results in 7 of the 52 patients without ascites ( $85 \%$ sensitivity, $87 \%$ specificity). Twenty-three of the 54 patients with perirectal and perivesical fat plan obliteration were identified by $\mathrm{CT}$ correctly, and 2 of the 55 patients without perirectal and perivesical fat plan obliteration had false-positive results on CT (43\% sensitivity and $94 \%$ specificity). CT was unable to detect any of the 20 patients who had diaphragmatic involvement. Also, 1 of the 81 patients without diaphragmatic involvement had false-positive results on CT. CT findings were not statistically significant for predicting diaphragmatic involvement. CT detected 15 of the 30 patients who had liver metastasis, However, it yielded false-positive results in 7 of the 77 patients without liver metastasis $(50 \%$ sensitivity, $91 \%$ specificity). Seven of the 22 patients with bladder metastasis were correctly identified by $\mathrm{CT}$, whereas 9 of the 80 patients without metastasis were found to have false-positive results on CT. CT findings were not statistically significant for predicting bladder metastasis. CT detected 22 of the 47 patients who had small and large bowel involvement, but it yielded false-positive results in 3 of the 47 patients without small and large bowel involvement (47\% sensitivity, $95 \%$ specificity). Twelve of the 49 patients with mesenteric involvement were detected by CT correctly, whereas 4 of the 52 patients without mesenteric involvement had false-positive results on CT. CT findings were not statistically significant for predicting mesenteric involvement. CT detected 9 of the 19 patients who had other metastases, and it yielded false-positive results in 13 of the 95 patients without other metastases (47\% sensitivity, $86 \%$ specificity). Eighty-eight of the 94 patients with adnexal mass could be detected with CT. However, CT yielded false-positive results in 3 of the 7 patients without

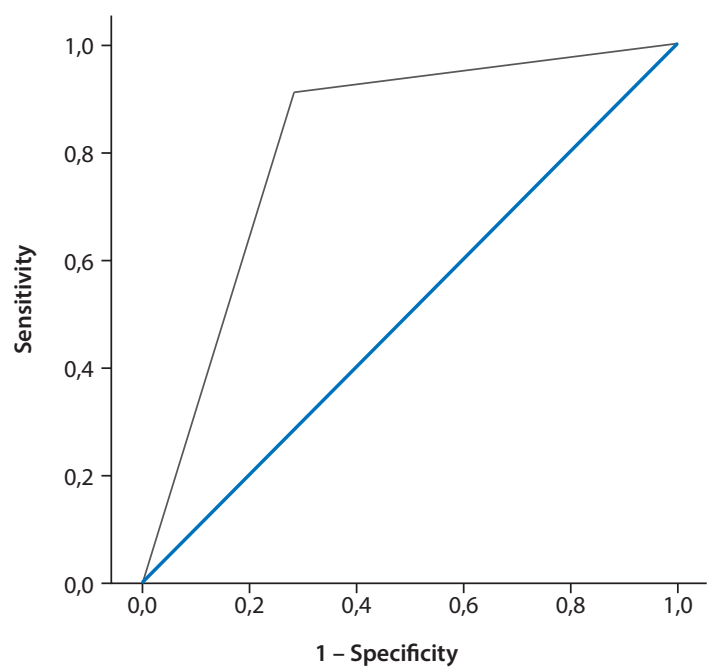

Figure 1. ROC curve of CT in detecting intraoperative findings

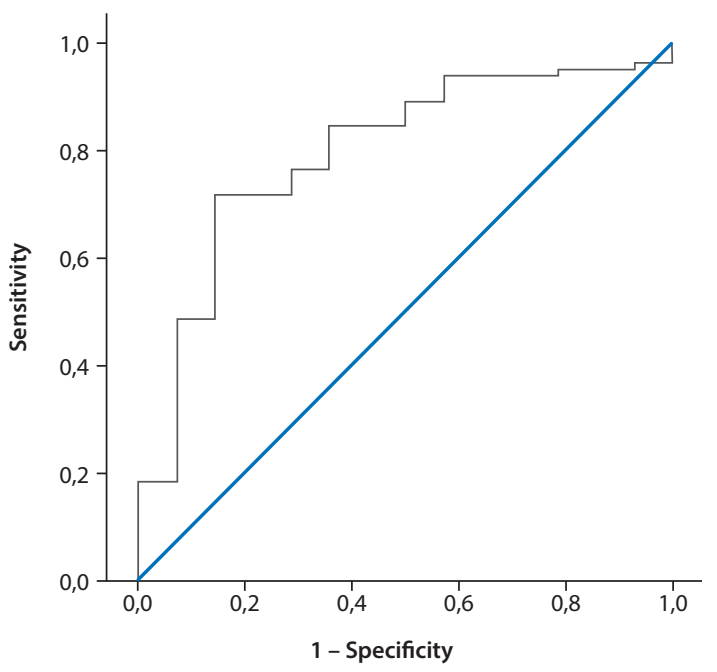

Figure 2. ROC curve of $C T$ in detecting intraoperative findings according to CA-125 (cut off value: 76.5 )

adnexal mass (sensitivity $94 \%$, specificity $70 \%$ ). When all parameters are considered, CT findings were statistically significantly correlated with intraoperative findings. Considering all parameters, the overall sensitivity and specificity of CT for detection of intraoperative findings were $91 \%$ and $71 \%$, respectively (Figure 1). At the determined cut off value of CA-125, the compliance of CT results with intraoperative findings was statistically significant. At CA-125 values of $>76.5 \mathrm{IU} / \mathrm{mL}, \mathrm{CT}$ could detect $79.8 \%$ of intraoperative findings (AUC) (Figure 2).

As far as the comparison of preoperative staging with surgical staging is concerned, we found that staging with CT was correct in 16 of the 23 surgically stage I patients, in 1 of the 4 surgically stage II patients, in 22 of the 47 surgically stage III patients, and in 16 of 38 surgically stage IV 


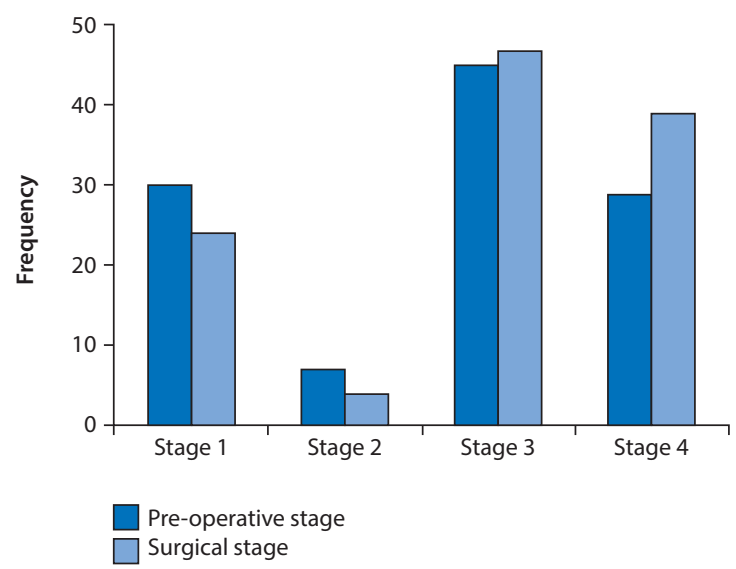

Figure 3. The distribution of preoperative and surgical stages

patients. The distribution of preoperative and surgical stages is shown in Figure 3. The total sensitivity of CT for staging was estimated at $49.1 \%$. When the patients were grouped as early (I and II) and late (III and IV) stages, sensitivity and specificity of preoperative staging with $\mathrm{CT}$ were calculated as $81 \%$ and $77 \%$, respectively.

In order to assess the reliability of preoperative CT for detection of potential complications of the surgery and additional interventions required, we analyzed preoperative CT findings based on complications occurring in GIS, GUS and CVS and requirement of additional surgeries. We detected a statistically significant correlation between CT results and intraoperative findings regarding small and large bowel involvement.

The analysis of GIS complications and the need of additional surgical procedures, in comparison with preoperative CT findings of GIS, detected a statistically significant correlation of CT findings related to small-large bowel and liver (superficial and parenchymal) involvement with intraoperative complications and requirement for additional surgical procedures (small and large bowel resection, liver resection) (AUC: 0.670, $\mathrm{p}<0.05$ ).

The analysis of CT findings related to GUS (uterine and tubal spread, bladder involvement, perirectal and perivesical fat plan obliteration), in comparison with GUS complications, found no statistically significant correlation between them, and none of the findings was predictive for the requirement of additional surgical procedures $(p>0.05)$.

In order to assess the predictability of potential complications of CVS, CT findings related to the retroperitoneal area were evaluated, and no statistically significant sensitivity was found $(p>0.05)$.

\section{DISCUSSION}

Surgical staging, as well as cytoreductive surgery, is critical as it affects the success of the treatment and prognosis [4,
12]. Determination of the extent of the disease preoperatively will allow to choose the best treatment strategy in clinical practice and identify the necessary surgical procedures in order to be prepared for them during the preoperative period. Due to reasonable costs and wide availability, CT is a commonly used imaging method in the preoperative evaluation, staging, and treatment planning of OC. However, it is insufficient to predict the correct stage as accuracy rates are reported between $50 \%$ to $92 \%$ in some series $[8,11,13]$. This may be due to the fact that CT evaluative capabilities for each anatomic region are different. Interpretation of CT findings would be more accurate if we were to know at which part of the examination it is more successful.

For the pelvis, a CT scan allows characterization of the adnexal mass and detection of the involvement of any surrounding organs (cervix, bladder, ureter, rectum, sigmoid, pelvic sidewall involvement). Regarding the upper abdominal region, involvement of the peritoneum, omentum, mesentery, small and large intestine, intra-extra parenchymal liver, diaphragm and retroperitoneal area can be detected by i.v. contrast CT scans [14]. However, it is a well-known fact that the most important limitation of $\mathrm{CT}$ in preoperative staging of $\mathrm{OC}$ is its inability to detect bowel surface, mesenteric, or peritoneal implants of $<5 \mathrm{~mm}$ reliably, especially in the absence of ascites $[15,16]$. In our study, the success rate of preoperative $\mathrm{CT}$ for prediction of postoperative surgical stage was estimated at $49 \%$.

To date, several studies have attempted to establish a predictive preoperative model to estimate successful tumor resectability, as well as to find predictors of suboptimal cytoreduction $[8,10,11,14]$. Fujwara et al., aimed to identify features of preoperative $\mathrm{CT}$ scans which can predict the outcomes of primary cytoreductive surgery in $\mathrm{OC}$ patients most successfully. They created 2 predictive models both including diffuse peritoneal thickening, infrarenal para-aortic or pelvic lymph node involvement, a bowel encasement tumor ( $\geq 2 \mathrm{~cm}$ ), and any tumor implants in the cul-de-sac. The accuracy rates of the models were $90.8 \%$ and $93.9 \%$ retrospectively [14]. For the same purpose, Bristow et al., identified 13 diagnostic features (peritoneal thickening, peritoneal implants $>2 \mathrm{~cm}$, small and large bowel mesenteric disease $>2 \mathrm{~cm}$, omental extension to stomach, spleen, or lesser sac, extension of the tumor to the pelvic sidewall/parametria/hydroureter, large-volume ascites, supra- and infrarenal lymph-adenopathy, diaphragm involvement, inguinal canal disease, liver lesions $>2 \mathrm{~cm}$, and porta hepatis/gallbladder disease) and calculated the predictive score to predict the chances of optimal cytoreduction. Using this model, the authors were able to predict the surgical outcome with a 93\% accuracy [17]. However, multi-institutional validation showed an accuracy of only $34 \%$ to $46 \%[5,14$, 17]. Unfortunately, as reported by several studies, it is not 
possible to predict with high accuracy which patients can be optimally debulked $[5,6]$.

In our study, when all parameters used for preoperative determination of the extent of the disease are taken into account, we observed that CT has a sensitivity of $91 \%$ and specificity of $71 \%$, a finding which is consistent with previous reports $[5,8,18]$. In addition, we found that CT could detect adnexal masses quite successfully, with a $93 \%$ sensitivity and a $70 \%$ specificity, as is consistent with previous reports, but it does not show sufficient sensitivity and specificity to determine local tumor dispersion like uterine and tubal involvement, pelvic fat plan obliteration and bladder metastases $[5,19,20]$. As it has been suggested before, we think MRI may yield more useful results regarding these areas [21,22].

CT findings were found to have high predictive value for the parameters of peritoneal involvement such as ascites, peritoneal carcinomatosis and omental involvement, but also it was found to be insufficient to detect peritoneal implants $<5 \mathrm{~mm}$, involvement of the intestinal surface and mesentery. It was concluded that CT did not have adequate sensitivity for these areas, as was pointed out in previous reports [15, 23, 24]. Again, as shown in many previous studies, CT had a low sensitivity for detection of retroperitoneal lymph node involvement and diaphragmatic metastasis [13]. We observed that CT had a 50\% sensitivity for detection of liver metastasis. Previous reports show great variability among different experienced radiologists regarding detection of liver metastasis [24].

Based on CT findings, the cut off value for serum CA125 level was calculated as $76.5 \mathrm{IU} / \mathrm{mL}$. Above this threshold, sensitivity and specificity of CT were calculated as $72.1 \%$ and $85.7 \%$, respectively ( $A \cup C=0.798$ ). CT findings were found to be statistically significantly correlated with intraoperative findings above this threshold ( $p=0.001, p<0.05$ ).

In order to assess the predictability of preoperative CT examination for detecting potential complications of the surgery and additional requirement of interventions, we analyzed preoperative CT findings based on the gastrointestinal system, genitourinary system, and cardiovascular system complications and additional surgeries. We detected a statistically significant correlation between CT findings related to the involvement of small and large bowels and liver (superficial and parenchymal) with intraoperative complications and additional surgical procedures (small and large bowel resection, liver resection).

The current study examined multiple radiographic features and clinical characteristics in an attempt to determine anatomical regions where $\mathrm{CT}$ is more successful in interpreting the findings more accurately. An important point of this study is that it was retrospective and thin-slice spiral multiplanar CT could not be used [25]. Also, the database incorporated the surgical results of different surgeons.
Accurate evaluation is an important prerequisite to select the best treatment strategy, to identify appropriate patients for surgery, and to determine specific surgical procedures preoperatively. Therefore, evaluation by an appropriate preoperative test is critical and prospective studies may be warranted.

\section{Conflict of interest}

The authors declare no competing interest.

No financial support received for this paper.

\section{REFERENCES}

1. Hennessy BT, Coleman RL, Markman M. Ovarian cancer. Lancet. 2009, 374, 1371-1382.

2. Ferlay J, Soerjomataram I, Dikshit R, [et al.]. Cancer incidence and mortality worldwide: sources, methods and major patterns in GLOBOCAN. Int J Cancer. 2012, 136, 359-386.

3. Trimbos JB, Vergote I, Bolis G, [et al.], EORTC-ACTION collaborators. European Organisation for Research and Treatment of Cancer-Adjuvant ChemoTherapy in Ovarian Neoplasm. Impact of adjuvant chemotherapy and surgical staging in early-stage ovarian carcinoma: European Organisation for Research and Treatment of Cancer-Adjuvant ChemoTherapy in Ovarian Neoplasm trial. J Nat/ Cancer Inst. 2003, 95, 113-125.

4. Vitale SG, Marilli I, Lodato M,Tropea A, Cianci A. The role of cytoreductive surgery in advanced-stage ovarian cancer: a systematic review. Updates Surg. 2013, 65, 265-270.

5. Axtell $A E$, Lee $M H$, Bristow $R E$, [et al.]. Multi-institutional reciprocal validation study of computed tomography predictors of suboptimal primary cytoreduction in patients with advanced ovarian cancer. J Clin Oncol. 2007, 25, 384-389.

6. Heudel PE, Selle F, Morice $P$, [et al.]. Initial management of advanced ovarian cancer: What radiological, pathological and surgical information are important for optimal therapeutic strategy? Bull Cancer. 2015, 102, 772-779.

7. Fischerova D. Ultrasound scanning of the pelvis and abdomen for staging of gynecological tumors: a review. Ultrasound Obstet Gynecol. 2011, 38, 246-266.

8. Glaser G, Torres M, Kim B, [et al.]. The use of CT findings to predict extent of tumor at primary surgery for ovarian cancer. Gynecol Oncol. 2013, 130, 280-283

9. Gąsiorowska E, Michalak M, WarchołW, [et al.]. Clinical application of HE4 and CA125 in ovarian cancer type I and type II detection and differential diagnosis. Ginekol Pol. 2015, 86 (2), 88-93.

10. Suidan RS, Ramirez PT, Sarasohn DM, [et al.]. A multicenter prospective trial evaluating the ability of preoperative computed tomography scan and serum CA-125 to predict suboptimal cytoreduction at primary debulking surgery for advanced ovarian, fallopian tube, and peritoneal cancer. Gynecol Oncol. 2014, 134, 455-461.

11. Kim HJ, Choi CH, Lee YY, [et al.]. Surgical outcome prediction in patients with advanced ovarian cancer using computed tomography scans and intraoperative findings. Taiwan J Obstet Gynecol. 2014, 53, 343-347. doi: 10.1016/j.tjog.2013.10.041.

12. Butler J, Gildea C, Poole J, [et al.]. Specialist surgery for ovarian cancer in England. Gynecol Oncol. 2015, doi: 10.1016/j.ygyno.2015.03.003.

13. Kitajima K, Murakami K, Yamasaki E, [et al.]. Diagnostic accuracy of integrated FDG-PET/contrast-enhanced CT in staging ovarian cancer: comparison with enhanced CT. Eur J Nucl Med Mol Imaging. 2008, 35, 1912-1920.

14. Fujwara K, Yoshino K, Enomoto T, [et al.]. Usefulness of computed tomography in predicting cytoreductive surgical outcomes for ovarian cancer. Arch Gynecol Obstet. 2011, 284, 1501-1507.

15. Pannu HK, Horton KM, Fishman EK. Thin section dual-phase multidetector-row computed tomography detection of peritoneal metastases in gynecologic cancers. J Comput Assist Tomogr. 2003, 27, 333-340.

16. Chandrashekhara SH, Thulkar S, Srivastava DN, [et al.]. Preoperative evaluation of peritoneal deposits using multidetector computed tomography in ovarian cancer. Br J Radiol. 2011, 84, 38-43.

17. Bristow RE, Duska L, Lambrou N, [et al.]. A model for predicting surgical outcome in patients with advanced ovarian carcinoma using computed tomography. Cancer. 2000, 89, 1532-1540. 
18. Shaaban A, Rezvani M. Ovarian cancer: detection and radiologic staging. Top Magn Reson Imaging. 2010, 21, 247-259.

19. Tsili AC, Tsampoulas C, Charisiadi A, [et al.]. Adnexal masses: accuracy of detection and differentiation with multidetector computed tomography. Gynecol Oncol. 2008, 110, 22-31.

20. Santoso JT, Robinson A, Suganda S, [et al.]. Computed tomography adnexal mass score to estimate risk for ovarian cancer. Arch Gynecol Obstet. 2014, 289, 595-600

21. Balan P. Ultrasonography, computed tomography and magnetic resonance imaging in the assessment of pelvic pathology. Eur J Radiol. 2006, 58, 147-155.
22. Lutz AM, Willmann JK, Drescher CW, [et al.]. Early diagnosis of ovarian carcinoma: is a solution in sight? Radiology. 2011, 259, 329-345.

23. Choi HJ, Lim MC, Bae J, [et al.]. Region-based diagnostic performance of multidetector $\mathrm{CT}$ for detecting peritoneal seeding in ovarian cancer patients. Arch Gynecol Obstet. 2011, 283, 353-360.

24. Akin O, Sala E, Moskowitz CS, [et al.]. Perihepatic metastases from ovarian cancer: sensitivity and specificity of CT for the detection of metastases with and those without liver parenchymal invasion. Radiology. 2008, 248, 511-517.

25. Rangarajan V. Three dimensional (3D) CT reconstruction in cancer imaging. Indian J Med Res. 2013, 137, 10-11. 